\title{
Isolated Tracheobronchial Amyloidosis
}

\author{
Madiha Mahfoudhi*, Imen Gorsane, Sami Turki, Taieb Ben Abdallah \\ Department of Internal Medicine A, Charles Nicolle Hospital, Tunis, Tunisia \\ Email: ${ }^{*}$ madiha mahfoudhi@yahoo.fr
}

Received 8 April 2015; accepted 14 September 2015; published 17 September 2015

Copyright (C) 2015 by authors and Scientific Research Publishing Inc.

This work is licensed under the Creative Commons Attribution International License (CC BY). http://creativecommons.org/licenses/by/4.0/

c) (i) Open Access

\begin{abstract}
The tracheobronchial amyloidosis is a rarely localized form. It may manifest as a cancer. Only histological results allow diagnosis confirmation. A 68-year-old man was admitted for exploration of a low abundance hemoptysis. The chest radiograph was normal. CT scan objectified thickening of the walls of the trachea and bronchi which are the seat of calcifications. Bronchoscopy found a burgeoning infiltrative lesion training the right side face of the trachea and the anterior edge of the hull. Histological results confirmed tracheobronchial amyloidosis. Typing of this amyloidosis concluded to AL form. The study of amyloid extension lesion was negative. Immunoelectrophoresis of proteins in the blood and urine was normal. The patient was sent to receive external beam radiation to prepare for endoscopic surgical removal. Post-operative suites were marked by a complete resolution of clinical signs particularly hemoptysis with a follow-up of two years.
\end{abstract}

\section{Keywords}

\section{Amyloidosis, Trachea, Biopsy}

\section{Introduction}

Amyloidosis is the extracellular deposition of amyloid substance having specific structure. Localized respiratory form of amyloidosis is extremely rare, particularly tracheobronchial site [1] [2].

Amyloidosis is not often taken into consideration in the differential diagnosis of patients with respiratory symptoms.

No clinical, biological and radiological feature is specific of the tracheobronchial localization [1]-[3].

Histological examination of a biopsy specimen guided by bronchoscopy confirms the diagnosis.

The immunohistochemical analysis allows for the typing of amyloidosis (AA, AL, ...) [1] [2].

The aim of this work is to review the scarcity of tracheobronchial amyloidosis, the diagnostic difficulty and the need for further monitoring because of the risk of transition to the systemic form and occurrence of

\footnotetext{
"Corresponding author.
} 
life-threatening complications.

\section{2-Case Report}

A 68-year-old man, with a history of a large smoking, had consulted for a low abundance hemoptysis.

The physical examination was without abnormalities particularly cardiac and pulmonary auscultation as well as neurological and abdominal examinations.

Biological investigations did not reveal anemia or inflammatory syndrome. The white blood cells count, the platelets count and the hemostasis tests were normal. The proteinuria was negative.

The chest radiograph was without anomalies.

Chest CT scan objectified thickening of the walls of the trachea with a moderate luminal narrowing and bronchi which are the seat of calcifications. Neither lymph node enlargement nor pleural effusion was found.

Bronchoscopy found a burgeoning infiltrative lesion training the right side face of the trachea and the anterior edge of the hull.

The hitological examination of tracheobronchial biopsy found an amyloidosis. Typing of this amyloidosis concluded to AL form.

The patient had no other visceral localization of amyloidosis implicating heart, kidney, bvgusalivary glands and bone marrow.

Immunoelectrophoresis of proteins in the blood and urine was normal.

The patient was sent to receive external beam radiation to prepare for endoscopic surgical removal.

Post-operative suites were marked by a complete resolution of clinical signs particularly hemoptysis with a follow-up of two years.

\section{Discussion}

The tracheobronchial amyloidosis is rare; about a hundred cases have been reported in the literature [2]-[4]. This is a localized form of amyloidosis [2] [3]. The most common amyloid pulmonary manifestations are tracheobronchial, nodular, and diffuse interstitial infiltrations.

It is usually symptomatically mimicking at many points bronchial neoplasia particularly when it occurs in a large smoking patient. It most frequently presents by coughing, wheezing, hemoptysis, or unexplained and recurrent pneumopathy [2]-[4].

The diagnosis is oriented by chest CT and confirmed by biopsy during bronchoscopy [2]-[5].

Neither chest CT results nor macroscopic aspects at bronchoscopy show specific signs.

The thickening aspect of the tracheal walls revealed in our case can be encountered in many diagnoses as carcinoma, lymphoma and sarcoidosis.

The clinical, radiological and endoscopic feature isn't contributive for diagnosis confirmation.

Only histological results showing amyloid deposits in the biopsied tissues allow confirming the diagnosis. Specific colorations are recommended to avoid missing the diagnosis.

Solitary nodules or elongated airway narrowing may be mistaken for neoplasia [6].

After confirming the diagnosis, investigations guided by the symptoms are necessary in search of systemic amyloidosis; cardiac and renal ultrasound evaluations are required to evaluate the patient prognosis [2]. Sometimes, many latent amyloid localizations can be revealed in the occasion of systematic research of other systemic involvement

In our case, tracheo-bronchial amyloidosis was isolated.

A bone marrow aspirate, Immunoelectrophoresis of proteins in the blood and urine are essential, in search of multiple myeloma.

Annual monitoring during the first ten years is essential since there's a possibility of conversion into systemic disease whose treatment differs from the localized form, almost progression to severe and permanent respiratory impairment and metastatic transformation may occur [6].

Rare similar cases to our patient were reported in the literature.

Brill AK et al. reported the case of a 65-year-old woman presenting recurrent bronchopulmonary infections, permanent cough and progressive dyspnea. The diagnosis of tracheobronchial amyloidosis was made by histological findings [6].

Yap JC presented a case of isolated tracheobronchial amyloidosis in a 72-year-old woman complaining of 
recurrent cough, dyspnoea, haemoptysis and respiratory infection. She received laser therapy with a good evolution [7].

The treatment of the localized tracheobronchial form is symptomatic.

The treatments offered in the localized tracheobronchial amyloidosis include external beam radiation, endoscopic procedures, debridement, removal and laser [6] [7].

Elongated stenosis can be managed by laser therapy, balloon dilatation or stenting.

Surgical treatment is rarely indicated. In case of localized AL (amyloid light chain) radiotherapy can be proposed in AL form [6].

Spontaneous regression has also been noted [8].

Bronchoscopic procedures and slow progression of this localized amyloidosis allow prognosis improvement. The prognosis is rarely worsened by the evolution to respiratory failure [2]-[4] [6] [8].

\section{Conclusion}

Localized amyloidosis such as tracheobronchial location, is rare and may be the first sign of a systemic disease, the pathogenesis remains unclear. Possible respiratory and systemic complications indicate a regular and prolonged surveillance.

\section{Disclosure Statement}

The authors have nothing to disclose.

\section{References}

[1] Georgiades, C.S., Neyman, E.G., Barish, M.A. and Fishman, E.K. (2004) Amyloidosis. A Review and CT Manifestations. Radiographics, 24, 405-416. http://dx.doi.org/10.1148/rg.242035114

[2] Cazalets, C., Belleguic, C., Sost, G., Caulet-Maugendre, S., Kernec, J., Droz, D. and Grosbois, B. (2002) TracheoBronchial Amyloidosis: Report of Two Cases. La Revue de Médecine Interne, 23, 317-321. http://dx.doi.org/10.1016/S0248-8663(01)00557-4

[3] Cazalets, C., Cadora, B., Mauduitb, N., Decaux, O., Ramée, M.-P., Le Pogamp, P., et al. (2003) Epidemiologic Description of Amyloidosis Diagnosed in University Hospital of Rennes from 1995 to 1999. La Revue de Médecine Interne, 24, 424-430. http://dx.doi.org/10.1016/S0248-8663(03)00136-X

[4] Racil, H., Ben Amar, J., Rouhou, S.C., Laaribi, G., Ayadi, A., Zarrouk, M., Chaouch, N. and Chabbou, A. (2011) Haemoptysis Revealing Tracheobronchial Amyloidosis. Revue de Pneumologie Clinique, 67, 109-112. http://dx.doi.org/10.1016/j.pneumo.2010.02.003

[5] Jiang, X. and Wang, G. (2012) On Primary Pulmonary Amyloidosis. European Journal of Radiology, 81, 1968. http://dx.doi.org/10.1016/j.ejrad.2011.04.005

[6] Brill, A.K., Woelke, K., Schädlich, R., Weinz, C. and Laier-Groeneveld, G. (2007) Tracheobronchial AmyloidosisBronchoscopic Diagnosis and Therapy of an Uncommon Disease: A Case Report. Journal of Physiology and Pharmacology, 58, 51-55.

[7] Yap, J.C., Wang, Y.T. and Poh, S.C. (1992) A Case of Primary Diffuse Tracheobronchial Amyloidosis Treated by Laser Therapy. Singapore Medical Journal, 33, 198-200.

[8] Hop, D.G. and Rasp, F.L. (1979) Spontaneous Regression of Tracheobronchial Amyloidosis. Chest, 76, $237-239$. 